\title{
The impact of environmental mycobiomes on geographic variation in COVID-19 mortality
}

\author{
Joshua Ladau*1, Katrina Abuabara*2,3, Angelica M. Walker ${ }^{4}$, Marcin P. \\ Joachimiak $^{5}$, Ishan Bansal ${ }^{1}$, Yulun $\mathrm{Wu}^{6}$, Elijah B. Hoffman ${ }^{6}$, Chaincy Kuo ${ }^{7}$, \\ Nicola Falco ${ }^{7}$, Jared Streich ${ }^{8}$, Mark J. van der Laan ${ }^{6}$, Haruko M. \\ Wainwright $^{7}$, Eoin L. Brodie ${ }^{7}$, Matthias $\mathrm{Hess}^{\dagger 9}$, Daniel Jacobson ${ }^{\dagger 8}$, and \\ James B. Brown ${ }^{\dagger 1,10,11}$ \\ ${ }^{1}$ Computational Biosciences Group, Lawrence Berkeley National Laboratory, Berkeley, CA, USA \\ 94720 \\ ${ }^{2}$ Division of Epidemiology and Biostatistics, University of California, Berkeley, CA 94720 \\ ${ }^{3}$ Department of Dermatology, University of California, San Francisco, CA 94115 \\ ${ }^{4}$ Bredesen Center for Interdisciplinary Research and Graduate Education, University of Tennessee \\ Knoxville, Knoxville, TN 37996 \\ ${ }^{5}$ Biosystems Data Science, EGSB, Lawrence Berkeley National Laboratory, 1 Cyclotron Rd., M/S \\ 74-316C, Berkeley, CA 94720 \\ ${ }^{6}$ Graduate Group in Biostatistics, University of California, Berkeley \\ ${ }^{7}$ Earth and Environmental Sciences Area, Lawrence Berkeley National Laboratory, 1 Cyclotron \\ Rd., M/S 74-316C, Berkeley, CA 94720 \\ ${ }^{8}$ Biosciences, Oak Ridge National Laboratory, Oak Ridge, TN 37830 \\ ${ }^{9}$ University of California, Davis, CA, USA \\ ${ }^{10}$ Statistics Department, University of California, Berkeley, CA, USA 94720 \\ ${ }^{11}$ Arva Intelligence, Inc., Salt Lake City, UT. 84101
}

\section{Abstract}

Mortality rates during the COVID-19 pandemic have varied by orders of magnitude across communities in the United States ${ }^{1}$. Individual, socioeconomic, and environmental factors have been linked to health outcomes of COVID-19 $9^{2,3,4,5}$. It is now widely appreciated that the environmental microbiome, composed of microbial communities associated with soil, water, atmosphere, and the built environment, impacts immune system development and

${ }^{*}$ Authors contributed equally

${ }^{\dagger}$ Authors contributed equally 
susceptibility to immune-mediated disease ${ }^{6,7,8}$. The human microbiome has been linked to individual COVID-19 disease outcomes ${ }^{9}$, but there are limited data on the influence of the environmental microbiome on geographic variation in COVID-19 across populations ${ }^{10}$. To fill this knowledge gap, we used taxonomic profiles of fungal communities associated with 1,135 homes in 494 counties from across the United States in a machine learning analysis to predict COVID-19 Infection Fatality Ratios (the number of deaths caused by COVID-19 per 1000 SARS-CoV-2 infections ${ }^{1}$; 'IFR'). Here we show that exposure to increased fungal diversity, and in particular indoor exposure to outdoor fungi, is associated with reduced SARS-CoV-2 IFR. Further, we identify seven fungal genera that are the predominant drivers of this protective signal and may play a role in suppressing COVID-19 mortality. This relationship is strongest in counties where human populations have remained stable over at least the previous decade, consistent with the importance of early-life microbial exposures ${ }^{11}$. We also assessed the explanatory power of 754 other environmental and socioeconomic factors, and found that indoor-outdoor fungal beta-diversity is amongst the strongest predictors of county-level IFR, on par with the most important known COVID-19 risk factors, including age $^{12}$. We anticipate that our study will be a starting point for further integration of environmental mycobiome data with population health information, providing an important missing link in our capacity to identify vulnerable populations. Ultimately, our identification of specific genera predicted to be protective against COVID-19 mortality may point toward novel, proactive therapeutic approaches to infectious disease.

\section{Introduction}

During the first eighteen months of the global COVID-19 pandemic, more than 176 million people were infected with SARS-CoV-2 wich resulted in 3.8 million deaths ${ }^{13,14}$. However, the toll of COVID-19 has varied greatly through both time and across geographical locations: for instance, case-fatality ratios across counties in the United States varied by over four orders of magnitude during the first eight months of the pandemic ${ }^{15,16,13}$. Although some of this variation can be explained by demographic, climatic, or social factors, other factors likely also contribute substantially to area-level variation in COVID-19 disease outcomes ${ }^{1,17,18}$. Identifying these other factors would be of value for forecasting trajectories of this and future pandemics, informing non-pharmaceutical interventions, and potentially indicating research directions towards novel therapeutic and immunological strategies.

In addition to interactions with pathogenic microbes that cause disease, humans interact constantly throughout their lives with a myriad of non-pathogenic microbes (e.g., bacteria and fungi) in the environment ${ }^{19,20}$. These interactions may significantly modulate disease outcomes - for instance, mitigating respiratory illnesses including asthma and allergic disease $^{21,7,22}$. While the mechanisms of action vary, beneficial effects of environmental microbes on health outcomes often share the following characteristics: ( $i$ ) the environmental microbes that have beneficial effects often originate from soils, freshwater environments, plants, and 
other non-anthropogenic sources, as opposed to the built environment, potentially reflecting a history of human evolutionary adaptation to them ${ }^{11,7,23,24,25}$; (ii) environmental microbes reduce disease severity by stimulating immune system development ${ }^{6,26,11}$; and (iii) exposure to a diversity of environmental microbes rather than a single taxon is necessary to confer beneficial effects ${ }^{7,23,25}$.

Functional immune response has been reported to be essential for moderating the severity of COVID-19 infection ${ }^{27,28}$. Prompted by the observations that the environmental microbiome influences immune system development and function ${ }^{6,29}$, and that COVID-19 severity correlates with hospital microbiome composition ${ }^{30}$ and with dysbiosis of the lung and ${ }^{31}$ the gut microbiome ${ }^{32,33}$, we hypothesized that the environmental microbiome, specifically the environmental mycobiome, is an important factor determining area-level variation in COVID-19 mortality.

\section{Results and Discussion}

To investigate the link between COVID-19 mortality and environmental fungi, we leveraged data on the taxonomic compositions of fungal communities from 1,135 homes across the United States (Figure E1) ${ }^{34,35}$ and data on COVID-19 from across the United States ${ }^{13,36}$. Each home had paired samples from indoors and outdoors, allowing comparison of the indoor and outdoor fungal communities. Using a novel approach, we also estimated SARS-CoV-2 IFR for each county across the United States, and consequently for each home for which fungal data were available (Supplementary Information). In addition, we extensively explored the influence of demographic, sociological, climate, and soil factors, some of which are known to influence IFR, fungal community composition, or both.

Most people in the United States interact primarily with microbes indoors, spending an average of $87 \%$ of their time in their homes and other constructed environments ${ }^{37}$. For fungi, in particular, important differences exist between primarily non-pathogenic outdoor species (which are sometimes also found indoors), and pathogenic or opportunistic species that proliferate in damp indoor environments ${ }^{38}$. Hence, we hypothesized that the presence of outdoor fungi in the built environment may be associated with reduced SARS-CoV-2 IFR. To test this hypothesis, we employed a two-tiered strategy. Firstly, we looked for association between relative fungal abundances (at the genus level) and SARS-CoV-2 IFR using a machine learning approach - iterative Random Forests (iRF). Secondly, we employed a quantile regression strategy to test the prediction that fungal indoor-outdoor beta diversity is most strongly associated with the upper quantiles of IFR - the most severely impacted counties.

The iRF analysis found marginal impact of the relative abundances of fungal genera or indoor-outdoor beta diversity after taking into account 754 demographic, sociological, climate, and soil factors (Supplementary Information). An ablation analysis revealed that after accounting for an expansive collection of other, alternative factors, the impact of fungal 
genera relative abundances had a statistically significant, albeit small $(\approx 1 \%)$ effect on mean IFR (Table E1, Figure E7). However, our quantile regression analysis revealed that the $75 \%$ and all quantiles above of SARS-CoV-2 IFR were strongly dependent on indoor-outdoor beta diversity. Indeed, indoor-outdoor beta-diversity is associated with reduced SARS-CoV-2 IFR (4.7 or fewer mortalities per 1000 infections; 90th percentile), while dissimilar indoor and outdoor communities (reduced indoor-outdoor beta diversity) are associated with elevated SARS-CoV-2 IFR (12.4 mortalities per 1000 infections; 90th percentile; Figure 1A-B). Hence, while the signal influencing mean IFR is relatively weak, the upper quantiles reveal strong and highly significant associations. These results remained qualitatively the same when the samples from each United States state and Census Division were individually omitted (Figures E2 - E4; Supplementary Information), indicating that the associations we detect are not driven by any single geographic region. Therefore, when accounting for the effects of confounding variables (below), high diversity of outdoor fungi present indoors appears to be associated with suppressed COVID-19 mortality, while lower diversity does not appear to confer these benefits.

Multiple lines of evidence indicate that the association between fungal beta-diversity and SARS-CoV-2 IFR suppression may be causal; that is, that the aforementioned associations are driven by the fungi themselves, rather than other confounding factors, such as demographic and climate variables. First, SARS-CoV-2 IFR early in the pandemic (April 2020 - November 2020) is positively associated with fungal indoor-outdoor beta-diversity - when pharmaceutical interventions were unavailable, but the association is weaker later in the pandemic (December 2020 - January 2021) when vaccination began and other pharmaceutical interventions were widely administered (Figure 1C). Second, the differences between indoor and outdoor fungal communities that are associated with SARS-CoV-2 IFR is explained primarily by soil edaphic factors - particularly $\mathrm{pH}$ - accounting for over $40 \%$ of the variance, while other factors that are known to be related to COVID-19, such as demographic and other non-climate factors, explain less than $30 \%$ of the variance combined (Figure 1D). This is intriguing, as it consistent with a causal model where soil edaphic factors affect COVID-19 severity by affecting microbial distributions, which are well-documented to be driven by soil characteristics, including $\mathrm{pH}^{39,40}$. Third, causal inference incorporating both fungal indooroutdoor beta-diversity, demographic, climate, and other predictors indicate a causal link between indoor-outdoor fungal beta-diversity and SARS-CoV-2 IFR even when these other potentially confounding variables are considered ( $p<0.001$, Supplementary Information).

To better understand the components of the fungal communities that contribute to the signal indicating suppression of SARS-CoV-2 IFR we employed a novel, beta-diversity correlation partitioning method (Supplementary Information). At least four of the following fungal genera play a key role in suppressing SARS-CoV-2 IFR: Alternaria, Aspergillus, Epicoccum, Eurotium, Toxicocladosporium, and Wallemia spp., and a novel Mycosphaerellaceae genus (some of these genera have correlated distributions making effects of individual genera indistinguishable; Figure 2A and 2C). High relative abundance of three of these genera both indoors and outdoors is necessary to detect SARS-CoV-2 IFR suppression: high relative 


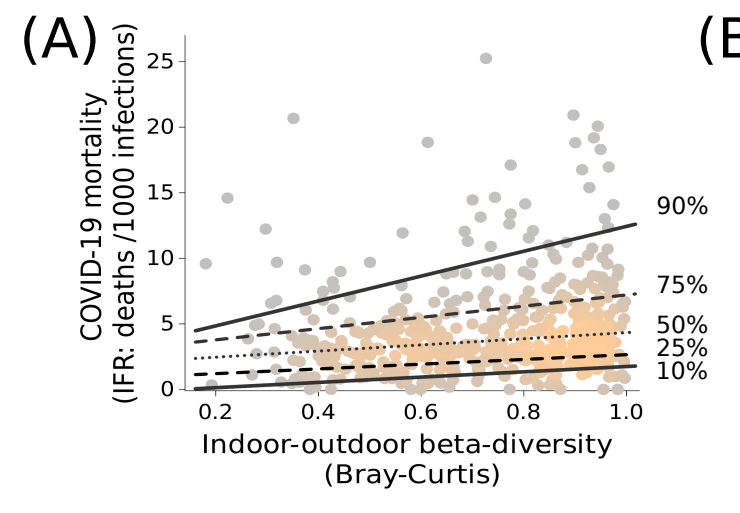

(C)

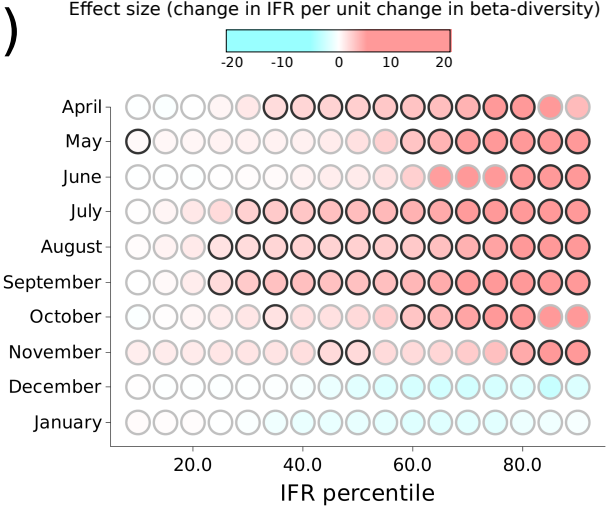

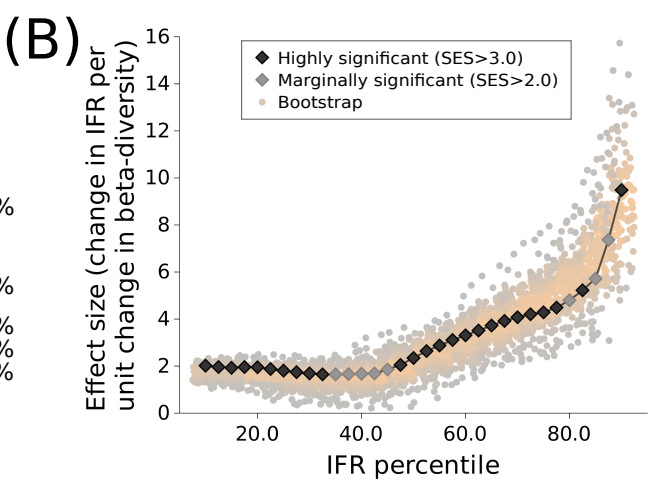

(D)

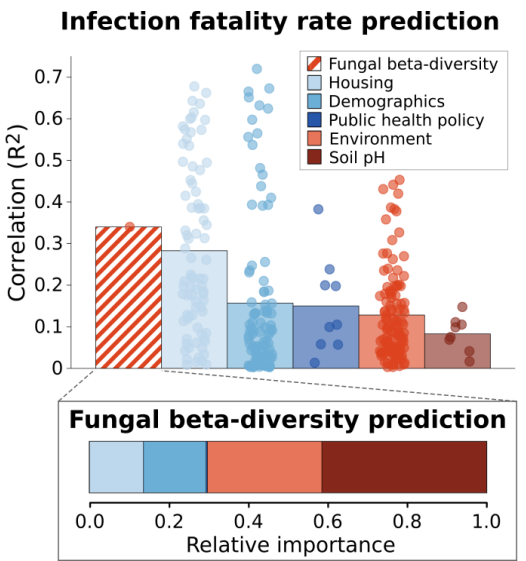

Figure 1: COVID-19 mortality is suppressed in homes where outdoor fungi occur indoors. (A) In United States homes where indoor and outdoor fungal communities are similar (low beta-diversity), COVID-19 mortality is reduced by over a factor of two compared to homes where the communities are dissimilar (high beta-diversity). Each point represents a United States county; shading indicates point density. Although the 90th percentile is sensitive to spatial autocorrelation, these trends are not driven by a single state or region of the United States (Figures E2 - E4). (B) The reductions in COVID-19 mortality (as measured by the standardized effect size, SES) are greatest in the upper quantiles of the COVID-19 mortality distribution, suggesting that outdoor fungi are sufficient but not necessary to reduce COVID-19 mortality. (C) The association between COVID-19 mortality and fungal betadiversity is strongest early in the pandemic, before December 2020 when vaccination began. Circles with black outlines indicate significant associations (SES> 3). (D) Fungal betadiversity is a strong predictor of suppression of SARS-CoV-2 IFR relative to other variables [column graph; points represent individual variables, columns show means; correlations give the association between the given variable and the windowed 75th percentile of IFR (see Supplementary Information)]. Moreover, fungal beta-diversity is most strongly associated with soil $\mathrm{pH}$ and other environmental variables (inset bar graph), suggesting that it is not a proxy for demographic and other variables that are known to effect COVID-19 mortality. 
abundance of Alternaria, Epicoccum, and Mycosphaerellaceae spp. just indoors - consistent with a primarily indoor origin - or outdoors - consistent with low indoor exposures - is insufficient (Figure 2B and Figure E5).

Our results pointing to beneficial effects of these seven genera for reducing COVID-19 mortality are novel. Different species from the same genus can have differential effects based on context, for example host genetics and age ${ }^{41}$, health status ${ }^{42}$, and interactions with other microbial exposures ${ }^{43}$. In many contexts, these genera are known to affect health negatively: for example, Alternaria, Wallemia, and Aspergillus spp. have been associated with increased rates of Irritable Bowel Syndrome, Ulcerative Colitis, and Keratitis, respectively ${ }^{44,45}$ (Table E2). Moreover, two species of Aspergillus can worsen outcomes of COVID-1944. However, environmental and endemic microbes may also reduce COVID-19 incidence and positively effect COVID-19 outcomes $^{46,47,48}$, and fungi from three aforementioned genera can also improve health outcomes for disease including oral cancer, Clostridium difficile-related diseases, and Seborrheic dermatitis, respectively ${ }^{44}$ (Table E2). The mechanism by which fungal elements may reduce infection risk could be through induction of trained immunity ${ }^{49,29,50}$. Fully understanding the context-dependence of the effects of environmental fungi on COVID-19 and other human disease outcomes is an area requiring further study.

In a general context, immune system development has been documented to be promoted by synergistic effects from the exposure to diverse microbes, as opposed to the effects of exposure to individual or a few microbes ${ }^{7,23,25}$. Consistent with these observations, the seven genera that we identified reveal positive synergistic effects: indoor-outdoor differences in relative abundance for genera individually tend to be poor predictors of SARS-CoV-2 IFR suppression (cross validation $R^{2}$ between 0 and 0.07 , median 0 ), while the beta-diversity of these genera taken together is strongly predictive (cross validation $R^{2}$ between 0.02 and 0.43 , median 0.19; Figure 2C).

We hypothesized the association between fungal diversity and SARS-CoV-2 IFR suppression will be strongest in regions where the human population has been stable for the last ten years or more, because people will have been more consistently exposed to the fungi in these regions ${ }^{6,11,51}$. Consistent with this prediction, in locations where the human population remained stable from 2010 to 2017, fungal beta diversity is more strongly associated with SARS-CoV-2 IFR suppression than in regions where it has fluctuated due to population turnover (Figure 3).

Collectively, these results accurately map locations where the occurrence of outdoor fungi in the indoor environment is forecast to most strongly suppress COVID-19 mortality in the United States: the Desert Southwest, Intermountain West, and Upper Midwest; a region that broadly corresponds with part of the country where soils are more alkaline, the primary predictor of indoor-outdoor fungal beta-diversity in our analysis (Figure 4). Soil pH is known to correlate with microbial diversity; it is also related to the water balance of ecosystems (mean annual precipitation relative to mean annual potential evapotranspiration) ${ }^{52}$, which in turn predicts fine dust aerosolization ${ }^{53}$. Thus along with climate ${ }^{32}, \mathrm{pH}$ may serve as a signature of microbial composition and propensity for microbial transport into homes via 


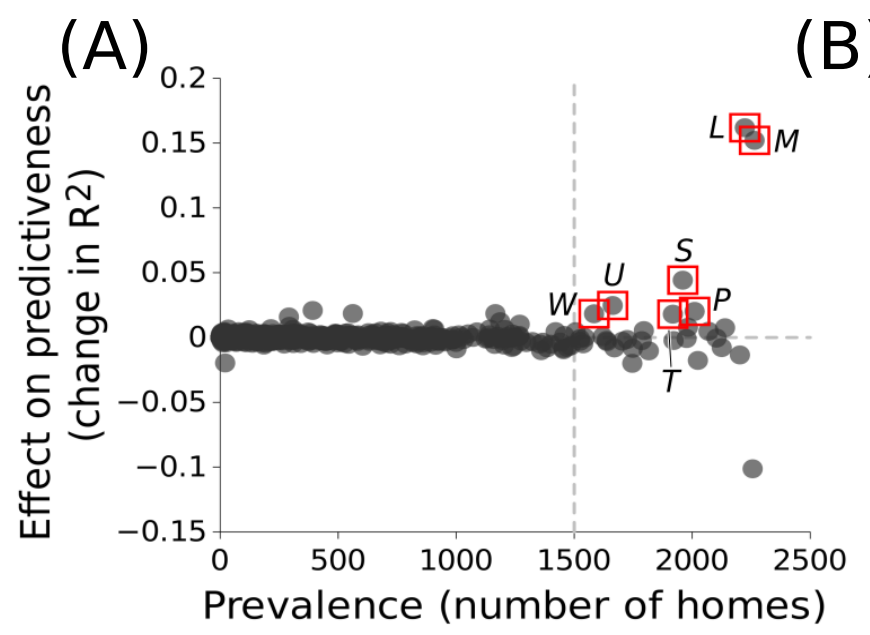

(C)
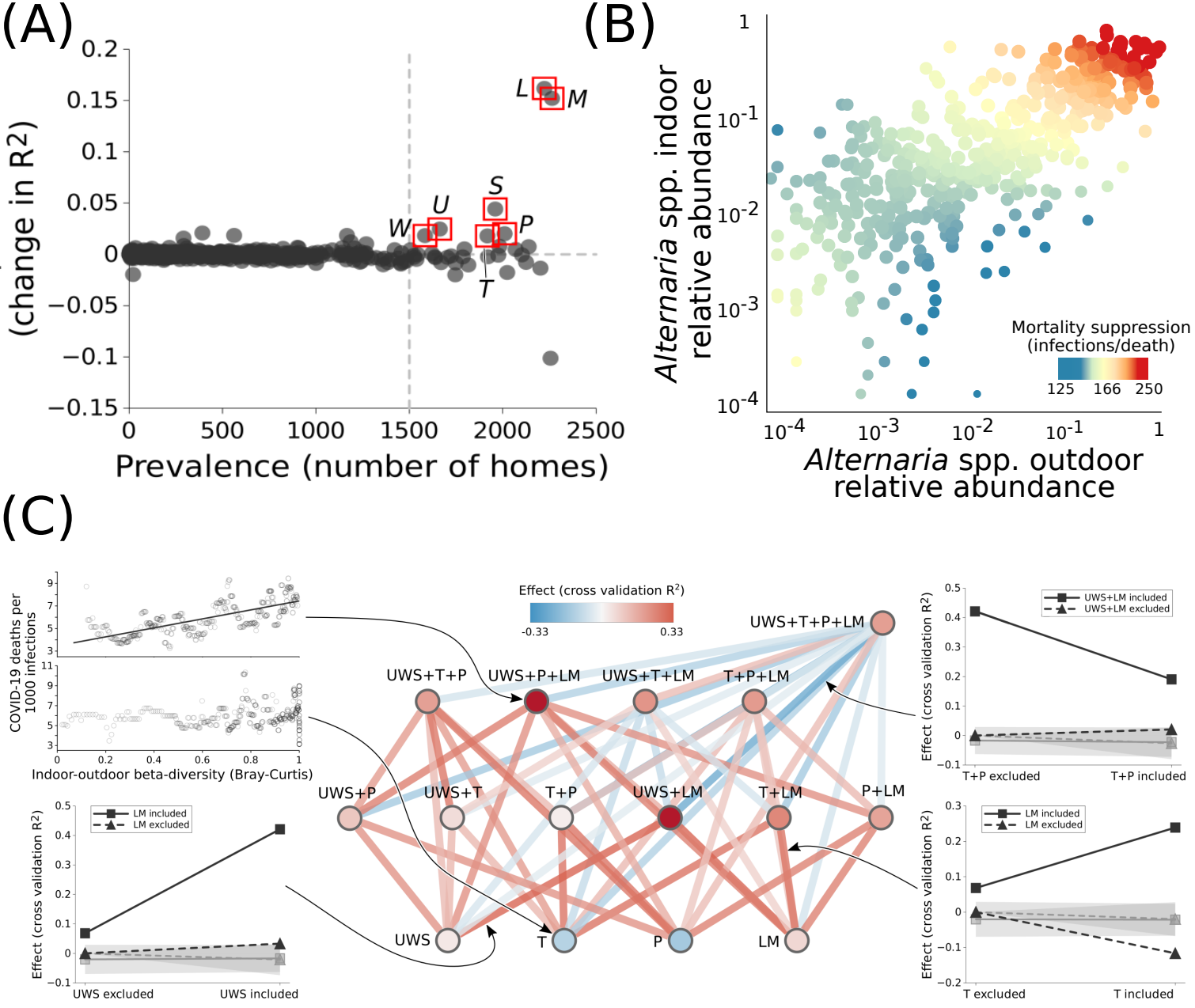

Figure 2: The occurrence of at least four outdoor fungal genera indoors suppresses COVID19 mortality. (A) Seven prevalent genera significantly increase the predictiveness of indooroutdoor fungal beta-diversity on COVID-19 mortality. Abbreviations are L: Alternaria, $S$ : Aspergillus, P: Epicoccum, U: Eurotium, T: Toxicocladosporium, W: Wallemia, M: Mycosphaerellaceae genus. (B) For some of these genera, including Alternaria spp., high relative abundance both outdoors and indoors is necessary for suppressed COVID-19 mortality. (C) However, the full beneficial effects result from synergistic effects of multiple genera: vertices represent sets of genera (colored by predictive power), while edges represent interactions when sets of genera are considered jointly as predictors (colored by interaction strength). Genera with correlated relative abundances are grouped together (e.g., LM: Alternaria and Mycosphaerellaceae genus). The graphs around the edges show examples of predictive power and interactions; gray lines demarcate null (randomized) expectations plus or minus one standard deviation. Except for when the full set of genera is considered (top right vertex), super-additive effects dominate, pointing to synergistic effects. 

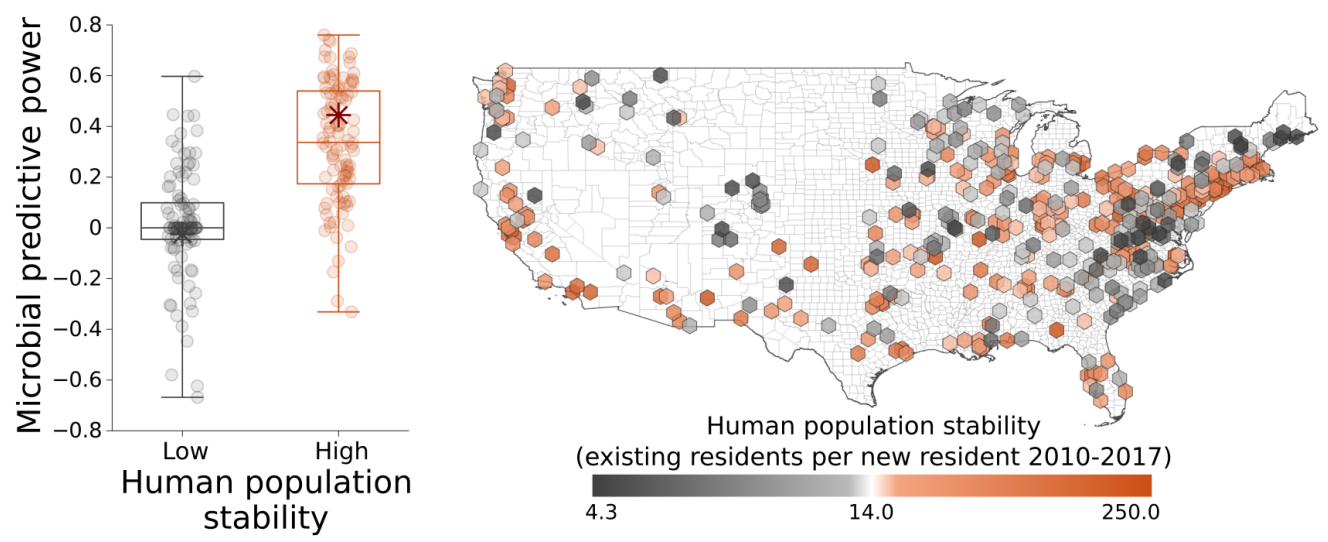

Figure 3: The occurrence of outdoor fungi indoors is more predictive of COVID-19 mortality in locations where people have been less transient from 2010-2017 than in regions where there are many new residents.

dust.

To reduce fatalities and limit the socioeconomic impact of the COVID-19 and future pandemics, a comprehensive understanding of factors affecting area-level variation in COVID-19 disease outcomes is essential ${ }^{5}$. We develop a novel strategy to address challenges in estimating SARS-CoV-2 IFR (as opposed to the case fatality ratio and other measures of COVID-19 severity), to identify factors driving SARS-CoV-2 IFR at large spatial scales. Here we find that environmental fungal communities, particularly indoor-outdoor beta diversity, are predictive of geographic variation in COVID-19 infection fatality ratios, above and beyond many other social and environmental factors. Our analyses indicate that exposure to high levels of outdoor fungi in homes is protective. If, as supported by our analysis, there is a causal relationship between long-term fungal exposures and SARS-CoV-2 IFR, then the environmental mycobiome constitutes an important missing link in our capacity to identify human populations that are vulnerable to poor outcomes from COVID-19. If, on the other hand, despite our extensive survey of environmental and socioeconomic predictors, we are missing as-yet unidentified confounding factors, our study underscores the utility of the environmental mycobiome as a biosensor ${ }^{54}$. Widespread beneficial effects of environmental fungi may not be specific to COVID-19; limited data support similar findings for childhood allergic disease and other viral diseases ${ }^{55,56,57}$, and it may be relevant for other autoimmune and immune-mediated diseases. Our results provide a foundation for research on the role of fungi and fungal interactions on the immune system, an important addition to a body of literature that has focused primarily on bacteria to date ${ }^{58,59}$. Recent advances in sequencing and classification of environmental fungi will enhance future efforts in this area ${ }^{60}$, and underscore the importance of biosurveillance for this and future pandemics. 
medRxiv preprint doi: https://doi.org/10.1101/2021.12.14.21267549; this version posted December 26, 2021. The copyright holder for this preprint (which was not certified by peer review) is the author/funder, who has granted medRxiv a license to display the preprint in perpetuity.

It is made available under a CC-BY-NC-ND 4.0 International license .

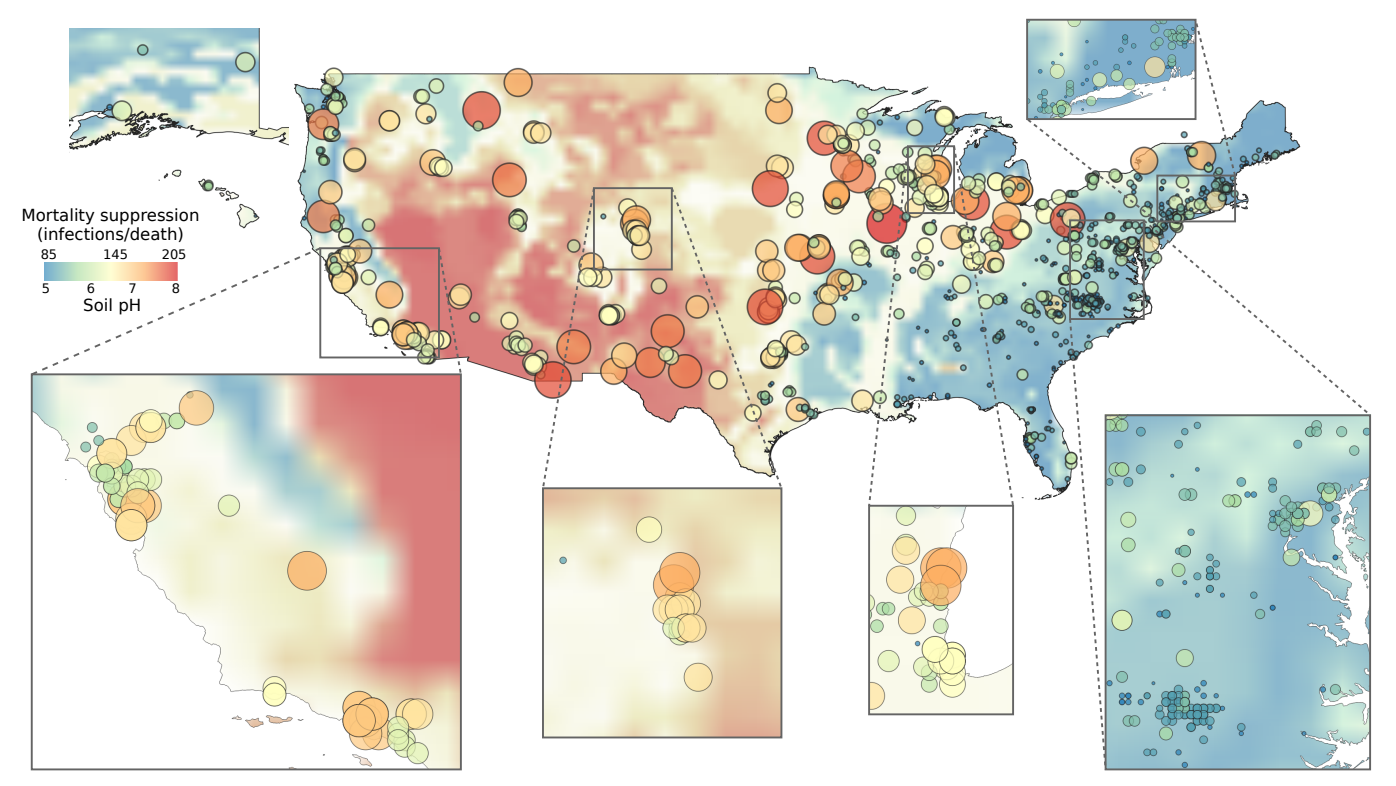

Figure 4: Fungal suppression of COVID-19 mortality varies regionally. In regions where soils tend to be basic (red background shading), indoor-outdoor fungal beta-diversity tends to be low, and fungal suppression of SARS-CoV-2 IFR is high (red dots). By contrast, the opposite trend holds in regions with acidic soils (blue background shading and dots); here, where fungal suppression of SARS-CoV-2 IFR is lessened, SARS-CoV-2 IFR can be high or low depending on whether other factors (e.g., climate, demographics) reduce SARS-CoV-2 IFR. 
medRxiv preprint doi: https://doi.org/10.1101/2021.12.14.21267549; this version posted December 26, 2021. The copyright holder for this preprint (which was not certified by peer review) is the author/funder, who has granted medRxiv a license to display the preprint in perpetuity.

It is made available under a CC-BY-NC-ND 4.0 International license .

\section{Methods}

Details of all methods are provided in Supplementary Information.

\section{Acknowledgements}

This manuscript has been coauthored by UT-Battelle, LLC under contract no. DE-AC0500OR22725 with the U.S. Department of Energy. The United States Government retains and the publisher, by accepting the article for publication, acknowledges that the United States Government retains a nonexclusive, paid-up, irrevocable, world-wide license to publish or reproduce the published form of this manuscript, or allow others to do so, for United States Government purposes. The Department of Energy will provide public access to these results of federally sponsored research in accordance with the DOE Public Access Plan (http://energy.gov/downloads/doe-public-access-plan, last accessed September 16, 2020). Work at Oak Ridge and Lawrence Berkeley National Laboratories was supported by the DOE Office of Science through the National Virtual Biotechnology Laboratory, a consortium of DOE national laboratories focused on response to COVID-19, with funding provided by the Coronavirus CARES Act, and was facilitated by previous breakthroughs obtained through the Laboratory Directed Research and Development Program of Lawrence Berkeley National Laboratory. M.P.J. was supported by a grant from the Laboratory Directed Research and Development (LDRD) Program of Lawrence Berkeley National Laboratory under U.S. Department of Energy Contract No. DE-AC02-05CH11231. Chris Mungall and Mark Miller (Lawrence Berkeley National Laboratory) assisted with mapping of Disbiome disease data to the Mondo ontology.

\section{Author Contributions}

J.L., J.B.B., K.A., M.P.J., M.H., Y.W., and I.B. made major contributions to writing the manuscript. J.L., J.B.B., K.A., M.P.J., M.H., Y.W., E.B.H., J.C.R., E.L.B., A.M.C., C.K., A.M.W., N.F., H.M.W., and D.J. designed this study. J.L., J.B.B., K.A., M.P.J., Y.W., E.B.H., I.B. J.C.R., A.M.W., A.M.C., and J.S. contributed analyses. J.B.B., J.L., D.J., and M.L. supervised students and managed analyses.

\section{Competing Interest Declaration}

The authors declare no competing interests. 


\section{Additional Information}

Supplementary Information is available for this paper. Correspondence and requests for materials should be addressed to Joshua Ladau.

\section{References}

[1] Klaus Desmet and Romain Wacziarg. Jue insight: Understanding spatial variation in covid-19 across the united states. Journal of Urban Economics, page 103332, 2021. ISSN 0094-1190. doi: https://doi.org/10.1016/j.jue.2021.103332. URL https://www.sciencedirect.com/science/article/pii/S0094119021000140.

[2] Rabail Chaudhry, George Dranitsaris, Talha Mubashir, Justyna Bartoszko, and Sheila Riazi. A country level analysis measuring the impact of government actions, country preparedness and socioeconomic factors on covid-19 mortality and related health outcomes. EClinicalMedicine, 25:100464, 2020. ISSN 2589-5370. doi: https://doi.org/10.1016/j.eclinm.2020.100464. URL https://www.sciencedirect.com/science/article/pii/S258953702030208X.

[3] Tamma Carleton, Jules Cornetet, Peter Huybers, Kyle C. Meng, and Jonathan Proctor. Global evidence for ultraviolet radiation decreasing covid-19 growth rates. Proceedings of the National Academy of Sciences, 118(1), 2021. ISSN 0027-8424. doi: 10.1073/pnas.2012370118. URL https : //www . pnas.org/content/118/1/e2012370118.

[4] COVID-19 Host Genetics Initiative. Mapping the human genetic architecture of covid19. Nature, 2021.

[5] John M McLaughlin, Farid Khan, Sarah Pugh, Frederick J Angulo, Heinz-Josef Schmitt, Raul E Isturiz, Luis Jodar, and David L Swerdlow. County-level predictors of coronavirus disease 2019 (covid-19) cases and deaths in the united states: What happened, and where do we go from here? Clinical Infectious Diseases, 11 2020. ISSN 1058-4838. doi: 10.1093/cid/ciaa1729. URL https://doi.org/10.1093/cid/ciaa1729. ciaa1729.

[6] Thomas Gensollen, Shankar S. Iyer, Dennis L. Kasper, and Richard S. Blumberg. How colonization by microbiota in early life shapes the immune system. Science, 352(6285):539-544, 2016. ISSN 0036-8075. doi: 10.1126/science.aad9378. URL https://science.sciencemag.org/content/352/6285/539.

[7] Markus J. Ege, Melanie Mayer, Anne-Cécile Normand, Jon Genuneit, William O.C.M. Cookson, Charlotte Braun-Fahrländer, Dick Heederik, Renaud Piarroux, and Erika von Mutius. Exposure to environmental microorganisms and childhood asthma. New England Journal of Medicine, 364(8):701-709, 2011. doi: 10.1056/NEJMoa1007302. URL https://doi.org/10.1056/NEJMoa1007302. PMID: 21345099. 
[8] Stephan P. Rosshart, Brian G. Vassallo, Davide Angeletti, Diane S. Hutchinson, Andrew P. Morgan, Kazuyo Takeda, Heather D. Hickman, John A. McCulloch, Jonathan H. Badger, Nadim J. Ajami, Giorgio Trinchieri, Fernando Pardo-Manuel de Villena, Jonathan W. Yewdell, and Barbara Rehermann. Wild mouse gut microbiota promotes host fitness and improves disease resistance. Cell, 171(5):10151028.e13, 2017. ISSN 0092-8674. doi: https://doi.org/10.1016/j.cell.2017.09.016. URL https://www.sciencedirect.com/science/article/pii/S0092867417310656.

[9] Shinya Yamamoto, Makoto Saito, Azumi Tamura, Diki Prawisuda, Taketoshi Mizutani, and Hiroshi Yotsuyanagi. The human microbiome and covid-19: A systematic review. PLOS ONE, 16(6):1-13, 06 2021. doi: 10.1371/journal.pone.0253293. URL https://doi.org/10.1371/journal.pone. 0253293.

[10] B. Brett Finlay, Katherine R. Amato, Meghan Azad, Martin J. Blaser, Thomas C. G. Bosch, Hiutung Chu, Maria Gloria Dominguez-Bello, Stanislav Dusko Ehrlich, Eran Elinav, Naama Geva-Zatorsky, Philippe Gros, Karen Guillemin, Frédéric Keck, Tal Korem, Margaret J. McFall-Ngai, Melissa K. Melby, Mark Nichter, Sven Pettersson, Hendrik Poinar, Tobias Rees, Carolina Tropini, Liping Zhao, and Tamara Giles-Vernick. The hygiene hypothesis, the covid pandemic, and consequences for the human microbiome. Proceedings of the National Academy of Sciences, 118(6), 2021. ISSN 0027-8424. doi: 10.1073/pnas.2010217118. URL https : //www . pnas . org/content/118/6/e2010217118.

[11] G. A. W. Rook, V. Adams, R. Palmer, L. Rosa Brunet, J. Hunt, and R. Martinelli. Mycobacteria and other environmental organisms as immunomodulators for immunoregulatory disorders. Springer Seminars in Immunopathology, 25(3-4):237-255, February 2004. doi: 10.1007/s00281-003-0148-9. URL https://doi .org/10.1007/s00281-003-0148-9.

[12] The OpenSAFELY Collaborative, Elizabeth Williamson, Alex J Walker, Krishnan Bhaskaran, Seb Bacon, Chris Bates, Caroline E Morton, Helen J Curtis, Amir Mehrkar, David Evans, Peter Inglesby, Jonathan Cockburn, Helen I McDonald, Brian MacKenna, Laurie Tomlinson, Ian J Douglas, Christopher T Rentsch, Rohini Mathur, Angel Wong, Richard Grieve, David Harrison, Harriet Forbes, Anna Schultze, Richard Croker, John Parry, Frank Hester, Sam Harper, Raf Perera, Stephen Evans, Liam Smeeth, and Ben Goldacre. Opensafely: factors associated with covid-19-related hospital death in the linked electronic health records of 17 million adult nhs patients. medRxiv, 2020. doi: 10.1101/2020.05.06.20092999. URL https://www . medrxiv. org/content/early/2020/05/07/2020.05.06.20092999.

[13] Ensheng Dong, Hongru Du, and Lauren Gardner. An interactive web-based dashboard to track covid-19 in real time. The Lancet infectious diseases, 20(5):533-534, 2020. 
[14] Ben Hu, Hua Guo, Peng Zhou, and Zheng-Li Shi. Characteristics of sars-cov-2 and covid-19. Nature Reviews Microbiology, pages 1-14, 2020.

[15] A Mazumder, M Arora, MS Sra, A Gupta, P Behera, M Gupta, M Agarwal, A Rao, SS Mohanta, GG Parameswaran, et al. Geographical variation in case fatality rate and doubling time during the covid-19 pandemic. Epidemiology \& Infection, 148, 2020.

[16] CDC Covid, Response Team, CDC COVID, Response Team, CDC COVID, Response Team, Stephanie Bialek, Virginia Bowen, Nancy Chow, Aaron Curns, et al. Geographic differences in covid-19 cases, deaths, and incidence - united states, february 12-april 7, 2020. Morbidity and Mortality Weekly Report, 69(15):465, 2020.

[17] Andrew C. Stokes, Dielle J. Lundberg, Irma T. Elo, Katherine Hempstead, Jacob Bor, and Samuel H. Preston. Covid-19 and excess mortality in the united states: A countylevel analysis. PLOS Medicine, 18(5):1-18, 05 2021. doi: 10.1371/journal.pmed.1003571. URL https://doi.org/10.1371/journal.pmed.1003571.

[18] Athanasios Damialis, Stefanie Gilles, Mikhail Sofiev, Viktoria Sofieva, Franziska Kolek, Daniela Bayr, Maria P. Plaza, Vivien Leier-Wirtz, Sigrid Kaschuba, Lewis H. Ziska, Leonard Bielory, László Makra, Maria del Mar Trigo, COVID19/POLLEN study group, and Claudia Traidl-Hoffmann. Higher airborne pollen concentrations correlated with increased sars-cov-2 infection rates, as evidenced from 31 countries across the globe. Proceedings of the National Academy of Sciences, 118(12), 2021. ISSN 0027-8424. doi: 10.1073/pnas.2019034118. URL https: //www . pnas.org/content/118/12/e2019034118.

[19] Danping Zheng, Timur Liwinski, and Eran Elinav. Interaction between microbiota and immunity in health and disease. Cell Research, 30(6):492-506, May 2020. doi: 10.1038/s41422-020-0332-7. URL https://doi.org/10.1038/s41422-020-0332-7.

[20] M. Hasan Mohajeri, Robert J. M. Brummer, Robert A. Rastall, Rinse K. Weersma, Hermie J. M. Harmsen, Marijke Faas, and Manfred Eggersdorfer. The role of the microbiome for human health: from basic science to clinical applications. European Journal of Nutrition, 57(S1):1-14, May 2018. doi: 10.1007/s00394-018-1703-4. URL https ://doi .org/10.1007/s00394-018-1703-4.

[21] Aaron Ver Heul, Joseph Planer, and Andrew L. Kau. The human microbiota and asthma. Clinical Reviews in Allergy \& Immunology, 57(3):350-363, November 2018. doi: 10.1007/s12016-018-8719-7. URL https://doi.org/10.1007/s12016-018-8719-7.

[22] Xi Fu, Zheyuan Ou, Mei Zhang, Yi Meng, Yanling Li, Jikai Wen, Qiansheng Hu, Xin Zhang, Dan Norbäck, Yiqun Deng, Zhuohui Zhao, and Yu Sun. Indoor bacterial, fungal and viral species and functional genes in urban and rural schools in shanxi province, china-association with asthma, rhinitis and rhinoconjunctivitis in high school students. 
Microbiome, 9(1):138, Jun 2021. ISSN 2049-2618. doi: 10.1186/s40168-021-01091-0. URL https://doi.org/10.1186/s40168-021-01091-0.

[23] Ilkka Hanski, Leena von Hertzen, Nanna Fyhrquist, Kaisa Koskinen, Kaisa Torppa, Tiina Laatikainen, Piia Karisola, Petri Auvinen, Lars Paulin, Mika J. Mäkelä, Erkki Vartiainen, Timo U. Kosunen, Harri Alenius, and Tari Haahtela. Environmental biodiversity, human microbiota, and allergy are interrelated. Proceedings of the National Academy of Sciences, 109(21):8334-8339, 2012. ISSN 0027-8424. doi: 10.1073/pnas.1205624109. URL https://www . pnas.org/content/109/21/8334.

[24] Michelle M. Stein, Cara L. Hrusch, Justyna Gozdz, Catherine Igartua, Vadim Pivniouk, Sean E. Murray, Julie G. Ledford, Mauricius Marques dos Santos, Rebecca L. Anderson, Nervana Metwali, Julia W. Neilson, Raina M. Maier, Jack A. Gilbert, Mark Holbreich, Peter S. Thorne, Fernando D. Martinez, Erika von Mutius, Donata Vercelli, Carole Ober, and Anne I. Sperling. Innate immunity and asthma risk in amish and hutterite farm children. New England Journal of Medicine, 375(5):411-421, 2016. doi: 10.1056/NEJMoa1508749. URL https://doi.org/10.1056/NEJMoa1508749. PMID: 27518660.

[25] Tari Haahtela, Stephen Holgate, Ruby Pawankar, Cezmi A Akdis, Suwat Benjaponpitak, Luis Caraballo, Jeffrey Demain, Jay Portnoy, and Leena von Hertzen. The biodiversity hypothesis and allergic disease: world allergy organization position statement. World Allergy Organization Journal, 6:3, 2013. doi: 10.1186/1939-4551-6-3. URL https://doi .org/10.1186/1939-4551-6-3.

[26] D. P. Strachan. Hay fever, hygiene, and household size. BMJ, 299(6710): 1259-1260, 1989. ISSN 0959-8138. doi: 10.1136/bmj.299.6710.1259. URL https ://www.bmj.com/content/299/6710/1259.

[27] Paul Bastard, Lindsey B Rosen, Qian Zhang, Eleftherios Michailidis, Hans-Heinrich Hoffmann, Yu Zhang, Karim Dorgham, Quentin Philippot, Jérémie Rosain, Vivien Béziat, et al. Autoantibodies against type i ifns in patients with life-threatening covid-19. Science, 370(6515), 2020.

[28] Qian Zhang, Paul Bastard, Zhiyong Liu, Jérémie Le Pen, Marcela Moncada-Velez, Jie Chen, Masato Ogishi, Ira K. D. Sabli, Stephanie Hodeib, Cecilia Korol, Jérémie Rosain, Kaya Bilguvar, Junqiang Ye, Alexandre Bolze, Benedetta Bigio, Rui Yang, Andrés Augusto Arias, Qinhua Zhou, Yu Zhang, Fanny Onodi, Sarantis Korniotis, Léa Karpf, Quentin Philippot, Marwa Chbihi, Lucie Bonnet-Madin, Karim Dorgham, Nikaïa Smith, William M. Schneider, Brandon S. Razooky, Hans-Heinrich Hoffmann, Eleftherios Michailidis, Leen Moens, Ji Eun Han, Lazaro Lorenzo, Lucy Bizien, Philip Meade, Anna-Lena Neehus, Aileen Camille Ugurbil, Aurélien Corneau, Gaspard Kerner, Peng Zhang, Franck Rapaport, Yoann Seeleuthner, Jeremy Manry, Cecile Masson, Yohann Schmitt, Agatha Schlüter, Tom Le Voyer, Taushif Khan, Juan Li, Jacques Fellay, Lucie 
Roussel, Mohammad Shahrooei, Mohammed F. Alosaimi, Davood Mansouri, Haya AlSaud, Fahd Al-Mulla, Feras Almourfi, Saleh Zaid Al-Muhsen, Fahad Alsohime, Saeed Al Turki, Rana Hasanato, Diederik van de Beek, Andrea Biondi, Laura Rachele Bettini, Mariella D'Angio', Paolo Bonfanti, Luisa Imberti, Alessandra Sottini, Simone Paghera, Eugenia Quiros-Roldan, Camillo Rossi, Andrew J. Oler, Miranda F. Tompkins, Camille Alba, Isabelle Vandernoot, Jean-Christophe Goffard, Guillaume Smits, Isabelle Migeotte, Filomeen Haerynck, Pere Soler-Palacin, Andrea Martin-Nalda, Roger Colobran, Pierre-Emmanuel Morange, Sevgi Keles, Fatma Çölkesen, Tayfun Ozcelik, Kadriye Kart Yasar, Sevtap Senoglu, Şemsi Nur Karabela, Carlos Rodríguez-Gallego, Giuseppe Novelli, Sami Hraiech, Yacine Tandjaoui-Lambiotte, Xavier Duval, Cédric Laouénan, COVID-STORM Clinicians, COVID Clinicians, Imagine COVID Group, French COVID Cohort Study Group, CoV-Contact Cohort, Amsterdam UMC Covid19 Biobank, COVID Human Genetic Effort, NIAID-USUHS/TAGC COVID Immunity Group, Andrew L. Snow, Clifton L. Dalgard, Joshua D. Milner, Donald C. Vinh, Trine H. Mogensen, Nico Marr, András N. Spaan, Bertrand Boisson, Stéphanie Boisson-Dupuis, Jacinta Bustamante, Anne Puel, Michael J. Ciancanelli, Isabelle Meyts, Tom Maniatis, Vassili Soumelis, Ali Amara, Michel Nussenzweig, Adolfo García-Sastre, Florian Krammer, Aurora Pujol, Darragh Duffy, Richard P. Lifton, Shen-Ying Zhang, Guy Gorochov, Vivien Béziat, Emmanuelle Jouanguy, Vanessa Sancho-Shimizu, Charles M. Rice, Laurent Abel, Luigi D. Notarangelo, Aurélie Cobat, Helen C. Su, and Jean-Laurent Casanova. Inborn errors of type i ifn immunity in patients with life-threatening covid19. Science, 370(6515), 2020. ISSN 0036-8075. doi: 10.1126/science.abd4570. URL https://science.sciencemag.org/content/370/6515/eabd4570.

[29] Mihai G. Netea, Jorge Domínguez-Andrés, Luis B. Barreiro, Triantafyllos Chavakis, Maziar Divangahi, Elaine Fuchs, Leo A. B. Joosten, Jos W. M. van der Meer, Musa M. Mhlanga, Willem J. M. Mulder, Niels P. Riksen, Andreas Schlitzer, Joachim L. Schultze, Christine Stabell Benn, Joseph C. Sun, Ramnik J. Xavier, and Eicke Latz. Defining trained immunity and its role in health and disease. Nature Reviews Immunology, 20(6):375-388, Jun 2020. ISSN 1474-1741. doi: 10.1038/s41577-020-0285-6. URL https : //doi .org/10.1038/s41577-020-0285-6.

[30] Clarisse Marotz, Pedro Belda-Ferre, Farhana Ali, Promi Das, Shi Huang, Kalen Cantrell, Lingjing Jiang, Cameron Martino, Rachel E. Diner, Gibraan Rahman, Daniel McDonald, George Armstrong, Sho Kodera, Sonya Donato, Gertrude Ecklu-Mensah, Neil Gottel, Mariana C. Salas Garcia, Leslie Y. Chiang, Rodolfo A. Salido, Justin P. Shaffer, Mac Kenzie Bryant, Karenina Sanders, Greg Humphrey, Gail Ackermann, Niina Haiminen, Kristen L. Beck, Ho-Cheol Kim, Anna Paola Carrieri, Laxmi Parida, Yoshiki Vázquez-Baeza, Francesca J. Torriani, Rob Knight, Jack Gilbert, Daniel A. Sweeney, and Sarah M. Allard. Sars-cov-2 detection status associates with bacterial community composition in patients and the hospital environment. Micro- 
biome, 9(1):132, Jun 2021. ISSN 2049-2618. doi: 10.1186/s40168-021-01083-0. URL https://doi .org/10.1186/s40168-021-01083-0.

[31] Haocheng Zhang, Jing-Wen Ai, Wenjiao Yang, Xian Zhou, Fusheng He, Shumei Xie, Weiqi Zeng, Yang Li, Yiqi Yu, Xuejing Gou, Yongjun Li, Xiaorui Wang, Hang Su, Zhaoqin Zhu, Teng Xu, and Wenhong Zhang. Metatranscriptomic Characterization of Coronavirus Disease 2019 Identified a Host Transcriptional Classifier Associated With Immune Signaling. Clinical Infectious Diseases, 73(3):376-385, 05 2020. ISSN 1058-4838. doi: 10.1093/cid/ciaa663. URL https://doi.org/10.1093/cid/ciaa663.

[32] Tao Zuo, Fen Zhang, Grace C.Y. Lui, Yun Kit Yeoh, Amy Y.L. Li, Hui Zhan, Yating Wan, Arthur C.K. Chung, Chun Pan Cheung, Nan Chen, Christopher K.C. Lai, Zigui Chen, Eugene Y.K. Tso, Kitty S.C. Fung, Veronica Chan, Lowell Ling, Gavin Joynt, David S.C. Hui, Francis K.L. Chan, Paul K.S. Chan, and Siew C. Ng. Alterations in gut microbiota of patients with COVID-19 during time of hospitalization. Gastroenterology, 159(3):944-955.e8, September 2020. doi: 10.1053/j.gastro.2020.05.048. URL https://doi.org/10.1053/j.gastro.2020.05.048.

[33] Longxian Lv, Silan Gu, Huiyong Jiang, Ren Yan, Yanfei Chen, Yunbo Chen, Rui Luo, Chenjie Huang, Haifeng Lu, Beiwen Zheng, Hua Zhang, Jiafeng Xia, Lingling Tang, Guoping Sheng, and Lanjuan Li. Gut mycobiota alterations in patients with covid19 and h1n1 infections and their associations with clinical features. Communications Biology, 4(1):480, Apr 2021. ISSN 2399-3642. doi: 10.1038/s42003-021-02036-x. URL https://doi .org/10.1038/s42003-021-02036-x.

[34] Albert Barberán, Joshua Ladau, Jonathan W. Leff, Katherine S. Pollard, Holly L. Menninger, Robert R. Dunn, and Noah Fierer. Continental-scale distributions of dustassociated bacteria and fungi. Proceedings of the National Academy of Sciences of the United States of America, 112(18):5756-5761, 2015. ISSN 00278424, 10916490. URL https://www . jstor.org/stable/26462658.

[35] Albert Barberán, Robert R. Dunn, Brian J. Reich, Krishna Pacifici, Eric B. Laber, Holly L. Menninger, James M. Morton, Jessica B. Henley, Jonathan W. Leff, Shelly L. Miller, and Noah Fierer. The ecology of microscopic life in household dust. Proceedings of the Royal Society B: Biological Sciences, 282(1814):20151139, September 2015. doi: 10.1098/rspb.2015.1139. URL https://doi.org/10.1098/rspb.2015.1139.

[36] The Atlantic. The covid tracking project, 2021. URL https://covidtracking.com.

[37] Neil E. Klepeis, William C. Nelson, Wayne R. Ott, John P. Robinson, Andy M. Tsang, Paul Switzer, Joseph V. Behar, Stephen C. Hern, and William H. Engelmann. The national human activity pattern survey (nhaps): a resource for assessing exposure to 
environmental pollutants. Journal of Exposure Science $\&$ Environmental Epidemiology, 11(3):231-252, Jul 2001. ISSN 1559-064X. doi: 10.1038/sj.jea.7500165. URL https://doi.org/10.1038/sj.jea.7500165.

[38] A. Nevalainen, M. Täubel, and A. Hyvärinen. Indoor fungi: companions and contaminants. Indoor Air, 25(2):125-156. doi: https://doi.org/10.1111/ina.12182. URL https://onlinelibrary.wiley.com/doi/abs/10.1111/ina.12182.

[39] Noah Fierer and Robert B. Jackson. The diversity and biogeography of soil bacterial communities. Proceedings of the National Academy of Sciences, 103 (3):626-631, 2006. ISSN 0027-8424. doi: 10.1073/pnas.0507535103. URL https : //www . pnas.org/content/103/3/626.

[40] Leho Tedersoo, Mohammad Bahram, Sergei Põlme, Urmas Kõljalg, Nourou S. Yorou, Ravi Wijesundera, Luis Villarreal Ruiz, Aída M. Vasco-palacios, Pham Quang Thu, Ave Suija, Miguel Rosas, Taavi Riit, David Ratkowsky, Karin Pritsch, Kadri Põldmaa, Meike Piepenbring, Cherdchai Phosri, Marko Peterson, Kaarin Parts, Kadri Pärtel, Eveli Otsing, Eduardo Nouhra, André L. Njouonkou, R. Henrik Nilsson, Luis N. Morgado, Jordan Mayor, Tom W. May, Luiza Majuakim, D. Jean Lodge, Su See Lee, Karl henrik Larsson, Petr Kohout, Kentaro Hosaka, Indrek Hiiesalu, Terry W. Henkel, Helery Harend, Liang dong Guo, Alina Greslebin, Gwen Grelet, Jozsef Geml, André De Kesel, Tan Dang, Xin Chen, Franz Buegger, Francis Q. Brearley, Gregory Bonito, Sten Anslan, Ra Abell, and Kessy Abarenkov. Global diversity and geography of soil fungi. Science, v. 346(no. 6213): pp. 1256688-1256688-2014 v.346 no.6213, Nov 2014. doi: 10.1126/science.1256688. URL https://doi.org/10.1126/science.1256688.

[41] M. J. Schuijs, M. A. Willart, K. Vergote, D. Gras, K. Deswarte, M. J. Ege, F. B. Madeira, R. Beyaert, G. van Loo, F. Bracher, E. von Mutius, P. Chanez, B. N. Lambrecht, and H. Hammad. Farm dust and endotoxin protect against allergy through a20 induction in lung epithelial cells. Science, 349(6252):1106-1110, September 2015. doi: 10.1126/science.aac6623. URL https ://doi.org/10.1126/science. aac6623.

[42] Melanie Schirmer, Sanne P. Smeekens, Hera Vlamakis, Martin Jaeger, Marije Oosting, Eric A. Franzosa, Rob ter Horst, Trees Jansen, Liesbeth Jacobs, Marc Jan Bonder, Alexander Kurilshikov, Jingyuan Fu, Leo A.B. Joosten, Alexandra Zhernakova, Curtis Huttenhower, Cisca Wijmenga, Mihai G. Netea, and Ramnik J. Xavier. Linking the human gut microbiome to inflammatory cytokine production capacity. Cell, 167(4):1125-1136.e8, November 2016. doi: 10.1016/j.cell.2016.10.020. URL https://doi.org/10.1016/j.cell.2016.10.020.

[43] Amy D Proal, Inge A Lindseth, and Trevor G Marshall. Microbe-microbe and hostmicrobe interactions drive microbiome dysbiosis and inflammatory processes. Discovery medicine, 23(124):51-60, 2017. 
[44] Yorick Janssens, Joachim Nielandt, Antoon Bronselaer, Nathan Debunne, Frederick Verbeke, Evelien Wynendaele, Filip Van Immerseel, Yves-Paul Vandewynckel, Guy De Tré, and Bart De Spiegeleer. Disbiome database: linking the microbiome to disease. BMC Microbiology, 18(1), June 2018. doi: 10.1186/s12866-018-1197-5. URL https://doi.org/10.1186/s12866-018-1197-5.

[45] Mycoses Study Group Education and Research Consortium. Doctor fungus, 2021. URL https://drfungus .org/.

[46] Richa B. Shah, Rachna D. Shah, Damien G. Retzinger, Andrew C. Retzinger, Deborah A. Retzinger, and Gregory S. Retzinger. Competing bioaerosols may influence the seasonality of influenza-like illnesses, including covid-19. the chicago experience. Pathogens, 10(9), 2021. ISSN 2076-0817. doi: 10.3390/pathogens10091204. URL https: //www.mdpi.com/2076-0817/10/9/1204.

[47] Andrew C. Retzinger and Gregory S. Retzinger. The role of surface in the pathogenesis and treatment of covid-19. COVID, 1(2):465-471, 2021. ISSN 2673-8112. doi: 10.3390/covid1020040. URL https : //www.mdpi.com/2673-8112/1/2/40.

[48] Manish Sagar, Katherine Reifler, Michael Rossi, Nancy S. Miller, Pranay Sinha, Laura F. White, and Joseph P. Mizgerd. Recent endemic coronavirus infection is associated with less-severe covid-19. The Journal of Clinical Investigation, 131(1), 1 2021. doi: 10.1172/JCI143380. URL https://www.jci.org/articles/view/143380.

[49] Anne Geller and Jun Yan. Could the induction of trained immunity by glucan serve as a defense against covid-19? Frontiers in Immunology, 11:1782, 2020. ISSN 1664-3224. doi: 10.3389/fimmu.2020.01782. URL https://www.frontiersin.org/article/10.3389/fimmu.2020.01782.

[50] Sadia Saeed, Jessica Quintin, Hindrik H. D. Kerstens, Nagesha A. Rao, Ali Aghajanirefah, Filomena Matarese, Shih-Chin Cheng, Jacqueline Ratter, Kim Berentsen, Martijn A. van der Ent, Nilofar Sharifi, Eva M. Janssen-Megens, Menno Ter Huurne, Amit Mandoli, Tom van Schaik, Aylwin Ng, Frances Burden, Kate Downes, Mattia Frontini, Vinod Kumar, Evangelos J. Giamarellos-Bourboulis, Willem H. Ouwehand, Jos W. M. van der Meer, Leo A. B. Joosten, Cisca Wijmenga, Joost H. A. Martens, Ramnik J. Xavier, Colin Logie, Mihai G. Netea, and Hendrik G. Stunnenberg. Epigenetic programming of monocyte-to-macrophage differentiation and trained innate immunity. Science, 345(6204):1251086, 2014. doi: 10.1126/science.1251086. URL https://www.science.org/doi/abs/10.1126/science.1251086.

[51] I Sadaf Farooqi and Julian M Hopkin. Early childhood infection and atopic disorder. Thorax, 53(11):927-932, 1998. ISSN 0040-6376. doi: 10.1136/thx.53.11.927. URL https://thorax.bmj.com/content/53/11/927. 
[52] E. W. Slessarev, Y. Lin, N. L. Bingham, J. E. Johnson, Y. Dai, J. P. Schimel, and O. A. Chadwick. Water balance creates a threshold in soil $\mathrm{pH}$ at the global scale. Nature, 540(7634):567-569, November 2016. doi: 10.1038/nature20139. URL https ://doi.org/10.1038/nature20139.

[53] P Achakulwisut, L J Mickley, and S C Anenberg. Drought-sensitivity of fine dust in the US southwest: Implications for air quality and public health under future climate change. Environmental Research Letters, 13(5):054025, May 2018. doi: 10.1088/17489326/aabf20. URL https://doi.org/10.1088/1748-9326/aabf 20.

[54] Maria Eugenia Inda and Timothy K. Lu. Microbes as biosensors. Annual Review of Microbiology, 74(1):337-359, 2020. doi: 10.1146/annurev-micro-022620-081059. URL https://doi .org/10.1146/annurev-micro-022620-081059. PMID: 32660390.

[55] Markus J. Ege, Melanie Mayer, Anne-Cécile Normand, Jon Genuneit, William O.C.M. Cookson, Charlotte Braun-Fahrländer, Dick Heederik, Renaud Piarroux, and Erika von Mutius. Exposure to environmental microorganisms and childhood asthma. New England Journal of Medicine, 364(8):701-709, 2011. doi: 10.1056/NEJMoa1007302. URL https://doi.org/10.1056/NEJMoa1007302.

[56] Melanie Schirmer, Sanne P. Smeekens, Hera Vlamakis, Martin Jaeger, Marije Oosting, Eric A. Franzosa, Rob ter Horst, Trees Jansen, Liesbeth Jacobs, Marc Jan Bonder, Alexander Kurilshikov, Jingyuan Fu, Leo A.B. Joosten, Alexandra Zhernakova, Curtis Huttenhower, Cisca Wijmenga, Mihai G. Netea, and Ramnik J. Xavier. Linking the human gut microbiome to inflammatory cytokine production capacity. Cell, 167 (4):1125-1136.e8, Nov 2016. ISSN 0092-8674. doi: 10.1016/j.cell.2016.10.020. URL https://doi.org/10.1016/j.cell.2016.10.020.

[57] Kei E. Fujimura, Alexandra R. Sitarik, Suzanne Havstad, Din L. Lin, Sophia Levan, Douglas Fadrosh, Ariane R. Panzer, Brandon LaMere, Elze Rackaityte, Nicholas W. Lukacs, Ganesa Wegienka, Homer A. Boushey, Dennis R. Ownby, Edward M. Zoratti, Albert M. Levin, Christine C. Johnson, and Susan V. Lynch. Neonatal gut microbiota associates with childhood multisensitized atopy and t cell differentiation. Nature Medicine, 22(10):1187-1191, Oct 2016. ISSN 1546-170X. doi: 10.1038/nm.4176. URL https://doi.org/10.1038/nm. 4176 .

[58] Junjie Qin, Ruiqiang Li, Jeroen Raes, Manimozhiyan Arumugam, Kristoffer Solvsten Burgdorf, Chaysavanh Manichanh, Trine Nielsen, Nicolas Pons, Florence Levenez, Takuji Yamada, Daniel R. Mende, Junhua Li, Junming Xu, Shaochuan Li, Dongfang Li, Jianjun Cao, Bo Wang, Huiqing Liang, Huisong Zheng, Yinlong Xie, Julien Tap, Patricia Lepage, Marcelo Bertalan, Jean-Michel Batto, Torben Hansen, Denis Le Paslier, Allan Linneberg, H. Bjørn Nielsen, Eric Pelletier, Pierre Renault, Thomas Sicheritz-Ponten, Keith Turner, Hongmei Zhu, Chang Yu, Shengting Li, Min Jian, Yan 
Zhou, Yingrui Li, Xiuqing Zhang, Songgang Li, Nan Qin, Huanming Yang, Jian Wang, Søren Brunak, Joel Doré, Francisco Guarner, Karsten Kristiansen, Oluf Pedersen, Julian Parkhill, Jean Weissenbach, Maria Antolin, François Artiguenave, Hervé Blottiere, Natalia Borruel, Thomas Bruls, Francesc Casellas, Christian Chervaux, Antonella Cultrone, Christine Delorme, Gérard Denariaz, Rozenn Dervyn, Miguel Forte, Carsten Friss, Maarten van de Guchte, Eric Guedon, Florence Haimet, Alexandre Jamet, Catherine Juste, Ghalia Kaci, Michiel Kleerebezem, Jan Knol, Michel Kristensen, Severine Layec, Karine Le Roux, Marion Leclerc, Emmanuelle Maguin, Raquel Melo Minardi, Raish Oozeer, Maria Rescigno, Nicolas Sanchez, Sebastian Tims, Toni Torrejon, Encarna Varela, Willem de Vos, Yohanan Winogradsky, Erwin Zoetendal, Peer Bork, S. Dusko Ehrlich, Jun Wang, and MetaHIT Consortium. A human gut microbial gene catalogue established by metagenomic sequencing. Nature, 464(7285):59-65, Mar 2010. ISSN 1476-4687. doi: 10.1038/nature08821. URL https://doi .org/10.1038/nature08821.

[59] Curtis Huttenhower, Dirk Gevers, Rob Knight, Sahar Abubucker, Jonathan H. Badger, Asif T. Chinwalla, Heather H. Creasy, Ashlee M. Earl, Michael G. FitzGerald, Robert S. Fulton, Michelle G. Giglio, Kymberlie Hallsworth-Pepin, Elizabeth A. Lobos, Ramana Madupu, Vincent Magrini, John C. Martin, Makedonka Mitreva, Donna M. Muzny, Erica J. Sodergren, James Versalovic, Aye M. Wollam, Kim C. Worley, Jennifer R. Wortman, Sarah K. Young, Qiandong Zeng, Kjersti M. Aagaard, Olukemi O. Abolude, Emma Allen-Vercoe, Eric J. Alm, Lucia Alvarado, Gary L. Andersen, Scott Anderson, Elizabeth Appelbaum, Harindra M. Arachchi, Gary Armitage, Cesar A. Arze, Tulin Ayvaz, Carl C. Baker, Lisa Begg, Tsegahiwot Belachew, Veena Bhonagiri, Monika Bihan, Martin J. Blaser, Toby Bloom, Vivien Bonazzi, J. Paul Brooks, Gregory A. Buck, Christian J. Buhay, Dana A. Busam, Joseph L. Campbell, Shane R. Canon, Brandi L. Cantarel, Patrick S. G. Chain, I-Min A. Chen, Lei Chen, Shaila Chhibba, Ken Chu, Dawn M. Ciulla, Jose C. Clemente, Sandra W. Clifton, Sean Conlan, Jonathan Crabtree, Mary A. Cutting, Noam J. Davidovics, Catherine C. Davis, Todd Z. DeSantis, Carolyn Deal, Kimberley D. Delehaunty, Floyd E. Dewhirst, Elena Deych, Yan Ding, David J. Dooling, Shannon P. Dugan, Wm Michael Dunne, A. Scott Durkin, Robert C. Edgar, Rachel L. Erlich, Candace N. Farmer, Ruth M. Farrell, Karoline Faust, Michael Feldgarden, Victor M. Felix, Sheila Fisher, Anthony A. Fodor, Larry J. Forney, Leslie Foster, Valentina Di Francesco, Jonathan Friedman, Dennis C. Friedrich, Catrina C. Fronick, Lucinda L. Fulton, Hongyu Gao, Nathalia Garcia, Georgia Giannoukos, Christina Giblin, Maria Y. Giovanni, Jonathan M. Goldberg, Johannes Goll, Antonio Gonzalez, Allison Griggs, Sharvari Gujja, Susan Kinder Haake, Brian J. Haas, Holli A. Hamilton, Emily L. Harris, Theresa A. Hepburn, Brandi Herter, Diane E. Hoffmann, Michael E. Holder, Clinton Howarth, Katherine H. Huang, Susan M. Huse, Jacques Izard, Janet K. Jansson, Huaiyang Jiang, Catherine Jordan, Vandita Joshi, James A. Katancik, Wendy A. Keitel, Scott T. Kelley, Cristyn Kells, Nicholas B. King, Dan Knights, Heidi H. Kong, Omry Koren, Sergey Koren, Karthik C. Kota, Christie L. 
Kovar, Nikos C. Kyrpides, Patricio S. La Rosa, Sandra L. Lee, Katherine P. Lemon, Niall Lennon, Cecil M. Lewis, Lora Lewis, Ruth E. Ley, Kelvin Li, Konstantinos Liolios, Bo Liu, Yue Liu, Chien-Chi Lo, Catherine A. Lozupone, R. Dwayne Lunsford, Tessa Madden, Anup A. Mahurkar, Peter J. Mannon, Elaine R. Mardis, Victor M. Markowitz, Konstantinos Mavromatis, Jamison M. McCorrison, Daniel McDonald, Jean McEwen, Amy L. McGuire, Pamela McInnes, Teena Mehta, Kathie A. Mihindukulasuriya, Jason R. Miller, Patrick J. Minx, Irene Newsham, Chad Nusbaum, Michelle O'Laughlin, Joshua Orvis, Ioanna Pagani, Krishna Palaniappan, Shital M. Patel, Matthew Pearson, Jane Peterson, Mircea Podar, Craig Pohl, Katherine S. Pollard, Mihai Pop, Margaret E. Priest, Lita M. Proctor, Xiang Qin, Jeroen Raes, Jacques Ravel, Jeffrey G. Reid, Mina Rho, Rosamond Rhodes, Kevin P. Riehle, Maria C. Rivera, Beltran RodriguezMueller, Yu-Hui Rogers, Matthew C. Ross, Carsten Russ, Ravi K. Sanka, Pamela Sankar, J. Fah Sathirapongsasuti, Jeffery A. Schloss, Patrick D. Schloss, Thomas M. Schmidt, Matthew Scholz, Lynn Schriml, Alyxandria M. Schubert, Nicola Segata, Julia A. Segre, William D. Shannon, Richard R. Sharp, Thomas J. Sharpton, Narmada Shenoy, Nihar U. Sheth, Gina A. Simone, Indresh Singh, Christopher S. Smillie, Jack D. Sobel, Daniel D. Sommer, Paul Spicer, Granger G. Sutton, Sean M. Sykes, Diana G. Tabbaa, Mathangi Thiagarajan, Chad M. Tomlinson, Manolito Torralba, Todd J. Treangen, Rebecca M. Truty, Tatiana A. Vishnivetskaya, Jason Walker, Lu Wang, Zhengyuan Wang, Doyle V. Ward, Wesley Warren, Mark A. Watson, Christopher Wellington, Kris A. Wetterstrand, James R. White, Katarzyna Wilczek-Boney, YuanQing Wu, Kristine M. Wylie, Todd Wylie, Chandri Yandava, Liang Ye, Yuzhen Ye, Shibu Yooseph, Bonnie P. Youmans, Lan Zhang, Yanjiao Zhou, Yiming Zhu, Laurie Zoloth, Jeremy D. Zucker, Bruce W. Birren, Richard A. Gibbs, Sarah K. Highlander, Barbara A. Methé, Karen E. Nelson, Joseph F. Petrosino, George M. Weinstock, Richard K. Wilson, Owen White, and The Human Microbiome Project Consortium. Structure, function and diversity of the healthy human microbiome. Nature, 486(7402):207-214, Jun 2012. ISSN 1476-4687. doi: 10.1038/nature11234. URL https://doi.org/10.1038/nature11234.

[60] R. Henrik Nilsson, Andy F. S. Taylor, Rachel I. Adams, Christiane Baschien, Johan Bengtsson-Palme, Patrik Cangren, Claudia Coleine, Heide-Marie Daniel, Sydney I. Glassman, Yuuri Hirooka, Laszlo Irinyi, Reda Iršènaitè, Pedro M. Martin-Sanchez, Wieland Meyer, Seung-Yoon Oh, Jose Paulo Sampaio, Keith A. Seifert, Frantisek Sklenář, Dirk Stubbe, Sung-Oui Suh, Richard Summerbell, Sten Svantesson, Martin Unterseher, Cobus M. Visagie, Michael Weiss, Joyce HC Woudenberg, Christian Wurzbacher, Silke Van den Wyngaert, Neriman Yilmaz, Andrey Yurkov, Urmas Kõljalg, and Kessy Abarenkov. Taxonomic annotation of public fungal its sequences from the built environment - a report from an april 10-11, 2017 workshop (aberdeen, uk). MycoKeys, 28:65-82, 2018. ISSN 1314-4057. doi: 10.3897/mycokeys.28.20887. URL https://doi.org/10.3897/mycokeys . 28.20887. 